\title{
Referee Acknowledgement for 2015
}

In this issue, we publish the names of those who reviewed manuscripts for us in 2015

The Editor-in-Chief, Specialist Editors and everyone involved in publishing BJC would like to extend our sincere thanks to them for contributing their expertise and time. Our referees play an invaluable role ensuring that BJC continues to publish the high quality original papers and reviews that make it one of the world's leading oncology journals.

Philippe A Cassier

Leon Aarons

Abdurrahman Abakay

BL Abbott

Gary Abel

Rinat Abramovitch

Anya Adair

Rosalind Adam

Richard Adams

Douglas Adamson

BB Aggarwal

Massimo Aglietta

Thomas Ahern

Nihal Ahmad

Atique U Ahmed

Ritva Ahomaki

Ariane Aigelsreiter

Jaffer Ajani

Suminori Akiba

Bilge Aktas

Ayodele A Alaiya

Peter Albers

Rita CR Albuquerque

Des Alcorn

Derek Alderson

Alain Algazi

$\mathrm{H}$ Ali

Shabbir Alibhai

Scheryll Alken

Khawla Al-Kuraya

Lucia Altucci

Robert Amato

Stefan Ambs

Martine Amiot

Lesley A Anderson

Laura Annovazzi

Shailendra Anoopkumar-Dukie

Amélie Anota

Lowell Anthony

Martina Anton

Alessandro Antonelli

Ahti Anttila

Yutaka Aoyagi

Giuseppe Aprile
Koji Arihiro

Hendrik-Tobias Arkenau

Saro H Armenian

Andrew Armstrong

Miranda EG Armstrong

Volker Arndt

Dirk Arnold

Joaquin Arribas

Corrado Asteria

Djordje Atanackovic

Wendy S Atkin

Riccardo Audisio

K Augoff

Michael J Ausserlechner

Philippe Autier

Anssi Auvinen

Peter Baade

Jean-Baptiste Bachet

Zsuzsanna Bago-Horvath

Shivani Bailey

Amanda Baker

Andrew E Balber

Albert Baldwin

Panagiotis Balermpas

Michaela S Banck

Udai Banerji

William E Barlow

Sandro Barni

Michele Barone

Sophie V Barrett

A Barzilai

David Basanta

Claudio Basilico

Sujit Basu

Veronique Bataille

Adrian Bateman

Eduard Battle

Michael Baum

Ian LP Beales

Alessandra Bearz

Nicole Beauchemin

Juergen Becker

Therese Becker

Muhammad Beg
Frederick Beland

Carine A Bellera

Sofiane Bendifallah

Charlotte Benson

Alice Berger

Walter Berger

Thierry Berghmans

Daniel Berney

Marie-Odile Bernier

Lori Bernstein

Alfredo Berruti

Dominik Berthold

Gaetano Bertino

Francesco Bertolini

Charles Betz

Charlotte Bevan

Krishnan Bhaskaran

Mohammad Saleem Bhat

Nirmala Bhoo Pathy

Fabrizio Bianchi

Siamak Bidel

Maurizio Bifulco

Andreas Bikfalvi

Mehmet Bilen

Ahmet Bilici

Alan Bilsland

Helgi Birgisson

Tithi Biswas

Benjamin Bitler

Peter C Black

Anneke Blackburn

Sarah Blagden

Tony Blakely

Giovanni Blandino

Roger Blanks

Maria Blettner

Judith Bliss

Thorarinn Blondal

Mark Bloomston

Simona Bo

Stefania Boccia

JA Bogaards

Ugo Boggi

L Bokhorst
C Richard Boland

Mark Bolding

Raffaella Bonecchi

Petri Bono

Stefanos Bonovas

Cecilia Bosco

Cristina Bosetti

Jacky Botterman

Judy C Boughey

Michel Bourguignon

Judith Bovée

Epie Boven

Anne Bowcock

Mark Bower

Norman F Boyd

Chiara Braconi

Angela Bradbury

Kathryn Bradbury

Penelope Bradbury

Gerard Brady

Etienne GC Brain

Kate Brain

Michael Bramble

Judith Brand

Yvonne Brandberg

Margie Brandwein-Gensler

Theodore Brasky

Shawn Bratton

Jeffrey J Brault

Michael Braun

Rod Braun

Jeremy Braybrooke

John Bridgewater

Nigel Brockton

Reuben Broom

Ewan Brown

Jennifer Brown

Robert Brown

Adam Brufsky

Deborah Watkins Bruner

Martin Brutsche

Daniel Buchanan

Tomas Buchler

Ronald Bukowski 
Anne-Laure Bulteau

JA Burgers

Lauren Averett Byers

Louise Cadman

Qiuyin Cai

Francesco Caiazza

Gregory Cairncross

Benjamin Cairns

Michael Caligiuri

George Calin

M Constanza Camargo

David Cameron

Christine Campbell

Kevin Camphausen

Francisco Jose Candido dos Reis

Nathan Cantoni

Maria D Cao

Yin $\mathrm{CaO}$

Vera Cappelletti

Federico Cappuzzo

Gabriele Capurso

Michele Carbone

Chris R Cardwell

Jennifer S Carew

Mariko Carey

Benedito A Carneiro

Ross Carruthers

William Carson

David Raul Francisco Carter

Brett S Carver

Oriol Casanovas

D Catovsky

Yvon E Cayre

Rachel M Ceballos

Jonathan Cebon

Daniela Cecconi

Yvonne Ceder

Paloma Cejas

Andres Cervantes

Jennifer Chan

Dhyan Chandra

David Chang

Shih-Ching Chang

Stephen J Chanock

Paul Chapman

Anil Chaturvedi

Ian Chau

James L Chen

Taosheng Chen

Yao-Tseng Chen

Young Koog Cheon

John Chester

Shakuntala Chhabra

Stephen K Chia

Maria Dolores Chiara

Anna Chiarelli

Shigeru Chiba

Martyn Chidgey

Julia Chisholm

Alexandre Chlenski

Yoon-La Choi

Irene Chong

Christos Chouaid

Mahavir Chougule

Alan Christie

Quincy Chu

Yu Jo Chua

Christine Chung

David Church

Udi Cinamon
Francois X Claret

GJ Clark

Robert Clarke

Stephen Clarke

Hope Clayton

Ellen Closs

Claude Cochet

Steven Cohen

Helen Coleman

William B Coleman

Helen M Coley

Marco Colleoni

Gary Collins

Peter Collins

Fiona Collinson

Marc Colonna

Bruce Colwell

Gerolama Condorelli

Stefan N Constantinescu

Alessandro Conti

Gary Cook

Natalie Cook

Colin S Cooper

Wendy Cooper

Domenico Coppola

Laurent Corcos

Pierpaolo Correale

Pippa Corrie

Richard J Cote

Nicholas Coupe

Vicky Coyle

Simon Crabb

E Crawford

S Michael Crawford

Danielle Crawley

Jenette Creaney

Ian Cree

Nicola Cresti

Rodney Croft

Deirdre P Cronin-Fenton

Emma Crosbie

Tom Crosby

Margaret E Cruikshank

Christopher Crum

Long Cui

Zoran Culig

Martine Culty

David Cunningham

Miroslava Cuperlovic-Culf

Anne Cust

Jack Cuzick

Daniel G Cyr

Rajvir Dahiya

Alv A Dahl

Björn Dahlbäck

Y Dai

Maria Grazia Daidone

Maria Giovanna Dal Bello

Luigino Dal Maso

Angus G Dalgleish

Fabio Dall'Olio

Ann Daly

Adam Dangoor

Daniel Danila

Laila Darwich

Timothy Daskivich

Adil Daud

Sarah E Daugherty

Matthew S Davids

John R Davies
Keith Davis

Noelle Davis

David W Dawson

Heather Dawson

Lesley Dawson

$R$ De Angelis

Richard De Boer

Elke De Bruyne

Nienke De Glas

Annelies De Klein

Harry De Koning

Ruggero De Maria

VDF De Mello

Michele De Palma

Silvia De Sanjose

Elisabeth GE De Vries

Olivier De Wever

Chris Deans

Anna DeFazio

Linda DeGraffenried

Patrice Delafontaine

Wendy Demark-Wahnefried

Jean-Baptiste Demoulin

Robert Den

Anant Desai

Mina Desai

Nandita M DeSouza

Jérôme Devy

Nandini Dey

Surbhi Dhar

Arunasalam Dharmarajan

Haryana Dhillon

Antonio Di Cristofano

Frederic Di Fiore

Giuseppe Di Lorenzo

Massimo Di Maio

Sérgio Dias

Concepcion Diaz-Arrastia

Adam Dicker

Maura N Dickler

Paul Dickman

Marie-Caroline Dieu-Nosjean

Analisa DiFeo

Peter W Dillon

Maurizio D'Incalci

Luc Yves Dirix

Sabina Dizdarevic

Alexander Dobrovic

Andrew Dodson

Val R Doherty

Laurent Dolle

Leslie Donaldson

Jixin Dong

Oliver Dorigo

Laure Dossus

Mitch Dowsett

Viji Draviam

Yvette Drew

Anatoly Dritschilo

Fran Duane

Dan G Duda

Peter Duesberg

Michael J Duffy

Stephen W Duffy

Mei Sheng Duh

Sarah Dumont

Amy Duncan

Malcolm Dunlop

Warwick Dunn

Fraser Duthie
Jonathan Dyke

Lars Dyrskjot

Martin Eatock

Karolina Edlund

R N Eisenman

Anders Ekbom

Karin Ekstrom-Smedby

E Eldering

Kevin M Elias

Elena Elimova

Matthew Ellis

Bassel El-Rayes

Wa'el El-Rifai

Charis Eng

Ellen Engelhardt

Zandra Ennis

Jonathan Epstein

Friederike Erdmann

Ayelet Erez

Rune Erichsen

Louise Eridsson

Morteza Esmaeili

Lluís Espinosa

D Gareth Evans

Muller Fabbri

Antongiullio Faggiano

Carole Fakhry

Marco Falasca

Stephen Falk

OO Faluyi

Francoise Farace

Matteo Fassan

André Fay

Roger Feakins

Kenneth CH Fearon

Liu Fei-Fei

Jaime Feliu

Martin E Fernandez-Zapico

Manuela Ferracin

Soldaro Ferrone

Patrizia Ferroni

Kate Fife

William Figg

Nicoletta Filigheddu

David Fine

Abe Fingerhut

Barbara Fingleton

Gaetano Finocchiaro

Marco Fiore

Matthew Firpo

Rebecca Fitzgerald

Patricia Fitzpatrick

David Flavell

Padraic Fleming

Steven Fleming

Olivia Fletcher

Ingrid Flight

Emmanouil Fokas

Gunnar Folprecht

Silvia C Formenti

Matthew Forshaw

Peter Forsyth

Orazio Fortunato

Theodoros Foukakis

Betsy Foxman

Adele Francis

Henrik Frederiksen

Wolf Fridman

Christine Friedenreich

Jan Frisell 
Clare Frobisher

Fieke Froeling

Peifen Fu

Robert Fuchs

Uwe Fuhr

T Fujita

Ada Funaro

Luc Furic

Sachio Fushida

Gilles Gadea

G Gaidano

Udo Gaipl

Timo Gaiser

David Gaist

Georgios Gakis

William M Gallagher

Eve Gallop-Evans

Apar Kishor Ganti

Patricia Ganz

Yuzhen Gao

Xavier Garcia del Muro

Montserrat Garcia-Closas

Jesus Garcia-Donas

C Garrido

Federico Garrido

Maria Luisa Gasparri

Pascal Gauduchon

Andrew Gaya

Ju Ian Geh

Jeanine Genkinger

Marco Gerlinger

Eugene Gerner

Andreas J Gescher

Paola Ghiorzo

Peter Gibbs

Todd M Gibson

Alexandra Gilbert

Duncan Gilbert

Mark R Gilbert

Graham Giles

Jonathan Gill

Andrew Gilmore

Tom Gilmore

Antonio Giordano

Paolo Giorgi Rossi

Elisa Giovannetti

Albert Girotti

Christian Gisselbrecht

David Gius

Rosalind Glasspool

Fergus Gleeson

Paul Glen

Joseph Gligorov

Annunziata Gloghini

Catherine Glynn

Rob Glynne-Jones

TE Godfrey

James J Going

Alisa Goldstein

David Goldstein

Marc Gollub

Barbara Gomes

Carlos Gomez-Martin

María del Carmen Gómez-Mateo

Roger Gomis

David Gonzalez de Castro

Jean-Marc Gornet

James Gossage

Noriko Gotoh

Heike Grabsch
Sergio A Gradilone

Janet Graham

Robert Graham

David Graves

Erin Greaves

Beverly B Green

Daniel Green

Heather Green

Jane Green

Michael Green

Victoria L Green

Mark Greene

Joel Greenson

Stefano Greggi

Alastair Greystoke

Sergey Grivennikov

Joanna Groden

AP Grollman

Kirsten Gronbaeck

Derek Grose

Milena Grossi

Rodrigo Guabiraba

Fernando Guerrero-Romero

C Guillen-Ponce

Roman Gulati

William J Gullick

Necdet Gunsoy

Marc Gunter

Rishein Gupta

Sumit Gupta

Laurel Habel

Oliver Hakenberg

Ruth Halaban

Carolyn Hall

Janet Hall

Per Hall

Jesper Hallas

Kiyohiro Hamatani

Stanley Hamilton

Yaniv Hamzany

Andrew Hanby

M Prakash Hande

Wedad Hanna

Torben Hansen

Reina Haque

James J Harding

Mark Harries

Adrian Harris

Holly Harris

Mark Harrison

Lauren Harshman

Kiyoshi Hasegawa

Sumaira Hasnain

Robert Hawkins

Nicholas Hayward

Hannelore Heemers

Uffe Heide-Jorgensen

Aslaug Helland

Charles Helsper

M E Hemler

Hiromichi Hemmi

Hugh Hemmings

Kari Hemminki

Helle Westergren Hendel

Amanda Herbert

Alexander G Heriot

Paul Hewitson

Dominique Heymann

Ralph Highnam

Mark Hill
Sharon Hillier

Nobuyoshi Hiraoka

Anita B Hjelmeland

Marko Hocevar

RC Hoeben

Gerald Hoefler

Lars Holmberg

Hubert Hondermarck

Naoko Honma

Elizabeth Hopper-Borge

Richard S Houlston

Sacha J Howell

Lynne Howells

Chun-Nan Hsu

Richard B Hubbard

Barry I Hudson

David Hui

Patrick Humbert

Damian Hussey

Cathy Hutchison

Georg Hutterer

Fortunata Iacopino

Daisuke Ichikawa

Shingo Ichimiya

Hisa Iinuma

Martin Illemann

David Ilson

Katsunori Imai

Frederico Innocenti

Pasquale Innominato

Hamish Ireland

Ruth Isherwood

Yasuhiro Ito

Michael Ittmann

Tomoo Iwakuma

Jose Luis Izquierdo Garcia

Erica L Jackson

Judith Jacobson

Martine J Jager

Anders Jakobsen

Alan James

Hay Janas

Yelena Janjigian

Tobias Janowitz

Eloisa Jantus-Lewintre

Aminah Jatoi

Gordon C Jayson

AH Jebar

Valerie Jenkins

Allan Jensen

Wen Jiang

Long R Jiao

Patrice Jichlinski

Irene Jillson

Masahisa Jinushi

Heikki Joensuu

Jonathan Joffe

Christoffer Johansen

Bruce Johnson

Colin Johnson

Kimberly J Johnson

Melissa L Johnson

Peter Johnson

Grace Johnston

Eric Jonasch

Jon G Jonasson

Resa M Jones

Robert Jones

Marjolijn Jongmans

Susan Jordan
David M Kaetzel

P Kameritsch

Michal Kaminski

Akihide Kamiya

S Kannan

Chryso Kanthou

Henry Kaplan

Premashis Kar

Matthias A Karajannis

Michael Karsy

Wouter Karthaus

Hidetaka Katabuchi

Ikuko Kato

David W Kaufman

Mehmet Kaynar

Sarah Keadle

Tom Keeley

Michael Keidar

Malcolm Kell

Caroline Kelly

Karl T Kelsey

Andrew Kennedy

Paraic Kenny

David Kerr

Rachel Kerr

Iftekhar Khan

Muhammad Khattak

Leeka Kheifets

Joan Khoo

Mandy Kiderlen

Emine Kilic

Anne E Kiltie

Eun-Kyung Kim

Isaac Kim

Reuben H Kim

Su-Jin Kim

Tae Il Kim

Randall J Kimple

Anne C Kirchhoff

Cari Kitahara

S Kitajima

Tohru Kiyono

Naomi Kiyota

Kristina Kjærheim

Jorg Kleeff

Alison Klein

Enno Klussmann

Jennifer Knox

Aung Ko Win

Andrew H Ko

Wayne Koch

Bogda Koczwara

Krithika Kodumudi

Alison B Kohan

Victor C Kok

Susanna Kola-Palmer

W Kolch

Viktor Kolzer

Natalia L Komarova

Anthony Kong

Lando LJ Koppes

Edward Korn

Alex Kostic

Michael Koukourakis

Adele Kraft

Oliver Krämer

Rebecca Kristeleit

Gunnar B Kristensen

Glen Kristiansen

Mary E Kroll 
Pieter L Kubben

Ragini Kudchadkar

Ernst Kuipers

Shiho Kuji

Addanki Kumar

Deepak Kumar

Satish Kumar

Hannah Kuper

Masato Kusunoki

Charlotte F Kweldam

Derek Kyte

Carlo La Vecchia

Uri Ladabaum

Jesper Lagergren

Luigi Laghi

Areti Lagiou

Pagona Lagiou

Robert W Laing

Barry J Laird

Philippe Lambin

Diether Lambrechts

Marene Landstrom

Rupert Langer

Ruth Langley

Cord Langner

C Lanvers-Kaminsky

James Larkin

Susanna C Larsson

Ulrik Lassen

Amy-Anne D Lassig

Arian Laurence

Sigurd Lax

Aljandro Lazo-Langner

Xuefen Le Bourhis

John Le Quesne

Michael Leahy

Jonathan Ledermann

Eunil Lee

Francis Y Lee

Jae-Lyun Lee

Jung-Min Lee

Simon Leedham

Max Leenders

Ulrich Lehmann

Celeste Leigh Pearce

Maarit Leinonen

Ferdy Lejeune

Riccardo Lencioni

MR Lennartz

Heinz Lenz

Yee Leung

Fabio Levi

Joan Lewis-Wambi

Timothy Ley

Dadong Li

Fengzhi Li

Jingmei Li

Paul V Licciardi

Jonathan D Licht

Emilia L Lim

Paul Limburg

Chia-Chin Lin

Zongming Lin

Annika Lindblom

Gerard J Linden

C Lingwood

Lance Liotta

Allan Lipton

Andrea A Lissoni

Saskia Litiere
Virginia Litle

Mark Little

Hongliang Liu

Hui-Wen Lo

Jeng-Fan Lo

Richard Lock

David Loeb

Fiona Lofts

Mignon Loh

Matthias Löhr

Martijn Lolkema

Dana P Loomis

Herbert Loong

Gilberto Lopes

Carlos López-Otín

Christopher Lord

Florian Lordick

Jan Lotvall

Emil Lou

Fotios Loupakis

Myriam Loyo

Binfeng Lu

Yunxia Lu

Michael Lübbert

Jay Lubin

DM Lubman

Anneke Lucassen

Alessandro Lugli

Juhua Luo

Philip Lupo

Manfred Lutz

Henry Lynch

Elsebeth Lynge

Georgios Lyratzopoulos

Jun Ma

Wen Wee Ma

Yuk Ting Ma

Donald Macarthur

Valentine Macaulay

Cristina Maccalli

Andrew MacDonald

Sara MacDonald

Laura M Machesky

Robert MacInnis

Jeffrey P MacKeigan

Vivienne MacLaren

Iain Macpherson

Srinivasan Madhusudan

Daichi Maeda

Kiyoshi Maeda

Chantal Magne Nde

Devalingam Mahalingam

Eamonn R Maher

John Maher

Nita Maihle

Tom Maishman

Patrick Maisonneuve

Ondrej Majek

Andreas Makris

Reza Malekzadeh

Carlo Maley

Hassan Malik

Nea Malila

David Malka

Annika Malmstrom

Mario Mandala

Henry Mandeville

Sridhar Mani

Roy Mano

Anthony Maraveyas
Paola Marcato

Giulio Marchesini

M Mareel

Jose Marin

Maurie Markman

Sharon Marsh

Diego R Martin

Jennifer Martin

Miguel Martin

Petra Martin

Tanimola Martins

Luca Mascitelli

Ralph Mason

Suleiman Massarweh

Lindsay Masson

Giuseppe Masucci

Keitaro Matsuo

Koji Matsuo

Tim Maughan

Nasim Mavaddat

Dimitris Mavroudis

Andrea Mazzarello

Karen McAdam

Christopher McCabe

Elaine McCartney

David J McConkey

Mary McCormack

Valerie McCormack

Paul McGale

Trevor McGoldrick

David McIntosh

Colin McKay

Ruth F McKee

Sharon McKenna

Fiona McKenzie

Donald C McMillan

Mairead McNamara

Joshua J Meeks

Akash Mehta

Stephanie Melkonian

Usha Menon

Alexander Menzies

Saiful Miah

Agnieszka Michael

Michael Michael

Dominique Michaud

Mark R Middleton

Diana Miglioretti

Wolfgang Mikulits

Anne Miles

David Miles

Anthony B Miller

Elizabeth Milne

Noel Milpied

Kosuke Mima

Koshi Mimori

Ahrum Min

Hironobu Minami

Andrew Minchinton

John Minna

Lopa Mishra

Katsuyuki Miura

Hiroshi Miyamoto

Eiji Miyoshi

Piergiorgio Modena

Esther Molina-Montes

Maurizio Mongiat

Alvaro N Monteiro

R Bruce Montgomery

Martine Moossdorff
Ana Maria Mora

Jurandyr Moreira de Andrade

Maria J Moreno

Carys Morgan

Eva Morris

Muriel Moser

Nicolas Mottet

Susan Moug

Kenneth Muir

Colin Muirhead

Somnath Mukherjee

Peter Mulders

Paul B Mullan

I Muller

Gabriele Multhoff

Teresita Muñoz-Antonia

Alastair Munro

Takashi Murakami

Ramachandran Murali

Peter Murchie

Jane Muret

Daniel Murphy

E Angela Murphy

Graeme Murray

Liam J Murray

Teemu J Murtola

Elizabeth Musgrove

Luciano Mutti

Louis Burt Nabors

Gabriele Nagel

Vinayak Nahar

Aung Naing

Raj Nair

Shigeki Nakagawa

Harikrishna Nakshatri

Steven A Narod

Paul Nathan

Joel W Neal

Andrea Necchi

Markus Neurath

Cindy Neuzillet

Charlotte Ngo

Shibani Nicum

Weiwei Nie

Forrest Nielsen

Hans Nijman

Katharina Nimptsch

Andrew Nixon

Simon Noble

Nijiro Nohara

Vikki G Nolan

Tobias Nordstrom

Nicola Normanno

Igor Novitzky-Basso

Gerard J Nuovo

Fiona Nussey

Lennarth Nystrom

Inge-Marie A Obdeijn

Alberto Ocana

Shuji Ogino

Karin Oien

Akinyemi Ojesina

Onyebuchi Okosiemeoe

Ivo Olivotto

Horst Olschewski

Aurelius Omlin

Takehisa Onishi

Adedayo Onitilo

Ruth O'Regan

Eileen O'Reilly 
Steffen Ormanns

Michael O'Rorke

Eigo Otsuji

Takemi Otsuki

Patrick Ott

Christian Ottensmeier

Michael J Overman

Babatunde O Oyajobi

Simon Pacey

David Page

Hannu Paimela

Tuya Pal

Jose Palacios

Emanuela Palmerini

Carlo Palmieri

Giusseppe Palmieri

Manoj Pandey

John Carl Panetta

Giuseppe Pannone

Sophie Papa

Irene Papatheodorou

Angelo Paradiso

Christos Paraskeva

Amadeo Parissenti

Elyse R Park

Hyung Seok Park

Upendra Parvathaneni

Patrizia Pasanisi

Elena Pasquale

Eddy Pasquier

Bhaumik Patel

Yogesh Patel

Ruth E Patterson

Chris L Paul

Jim Paul

Patricia Pautier

Patrick Pauwels

Yudi Pawitan

Miranda Payne

Mick Peake

Anette Pedersen

Frank Peinemann

Claudio Pelucchi

Louis Pelus

Nicolas Penel

Kathryn L Penney

George Pentheroudakis

Silvia Penuela

Paola Perego

Christos Perisanidis

Claire M Perks

Eleni Petridou

Russell Petty

Ludovic Peyre

Roger Phillips

Maria Carmela Piccirillo

Martin Pichler

Jean-Yves Pierga

Geraldine Pignot

James Pine

Paolo Pinton

Munir Pirmohamed

Xavier Pivot

Barry Pizer

Rina Plattner

Chris Plummer

Elizabeth Plummer

M Pocard

John Poirier

Jason Pole
Venerino Poletti

Marina Pollan

Richard Pomerantz

Elizabeth Poole

Sanjay Popat

Cornel Popovici

Camillo Porta

G Portella

Michael Postow

Anton Pottegård

Vanessa Potter

Vasiliki Poulaki

Desmond Powe

Danielle Power

Gerald Prager

KV Prasanth

Hans Prenen

Timothy Price

John N Primrose

Kathy Pritchard-Jones

David Propper

Elena Provenzano

Fabio Puglisi

Eero Pukkala

Cornelis JA Punt

Martine Puts

Lu Qi

Lei Quan

Gaelle Quereux

Charles M Quick

Julie A Quinlivan

Morten Quist

Milan Radovich

Estelle Rage

Sheela Rao

Ramandeep Rattan

Avraham Raz

Daniel Rea

Vinciane Rebours

Andrés Redondo

Damon Reed

Michael Reedijk

Tarik Regad

Reuven Reich

Cary Reid

Glen Reid

Ofer Reizes

Marc Remacle

Sabatier Renaud

Jerry M Rice

Danny Rischin

Michael Risk

Christoph A Ritter

Christos Rizos

Kathryn Robb

Simon P Robinson

Andrew W Roddam

Gary Rodin

Oluf Dimitri Røe

Einar K Rofstad

Sabine Rohrmann

Camilo Rojas

Joseph Roland

Eve Roman

MF Romano

Iris L Romero

Martin M Root

Rafael Rosell

Paul J Ross

Valentina Rossi
Vittorio Rosti

Kai Rothkamm

Sven Rottenberg

Jeff Round

Campbell Roxburgh

Steve Rozen

Domenico Rubello

IT Rubio

Marek Ruchala

Charles Rudin

Anja Rudolph

Eliana Rulli

Antonio Russo

Mark Rutherford

Leonard Rybak

Adrian Sacher

Siegal Sadetzki

Corinna Sadlier

Xavier Sagaert

Surinder Sahota

Evis Sala

Ramon Salazar

Helga Salvasen

Goli Samimi

Wolfram Samlowski

Josemir W Sander

M Santibanez

Alessandro Santin

Daniele Santini

Matteo Santoni

Outi R Saramaki

Tytti Sarkeala

Amod A Sarnaik

Peter Sasieni

Hiroaki Satoh

Philippa TK Saunders

Alexandru Saveanu

Ben Saville

David Sawbridge

Elinor Sawyer

Stefania Scala

Mario Scartozzi

Louise Scheen

Werner Scheithauer

Peter J Schmidt

Marc Schmidt-Supprian

Manfred Schmitt

Hans-Joachim Schmoll

Helena Schock

Patrick Schöffski

Leo Schouten

HM Schuller

Anke Schutz

Margaret Schwarz

Heidi Schwarzenbach

Francesco Sclafani

Halden F Scott

Kenneth Scott

David Sebag-Montefiore

E Segelov

Christof Seidl

Arnaud Seigneurin

Peter Senter

Ben Seon

Beatriz Serrano

Thomas Seufferlein

Alessandro Sgambato

Manish Shah

Ricky Sharma

Rohini Sharma
Reuben Shaw

Richard Shaw

Noula Shembade

Bairong Shen

Jessica Sheringham

Chanjuan Shi

Meredith Shiels

Hideaki Shimada

Daniel Shin

Eiji Shinto

Tanri Shiozawa

RE Shore

Susan Short

Neal Shukla

Robert Siddaway

Michele Signore

Andrew Silver

Gerard Silvestri

Ricard Simo

Michael Simon

Susanne Singer

Ajay Singh

Pankaj Singh

Rakesh Singh

Siddharth Singh

Marianne Sinn

Roderick Skinner

Kristin Skougaard

Nicholas Skuli

Susan L Slager

Katherine Sleeman

Jeremy S Slone

Keiran Smalley

Charles Smith

Martyn Smith

Steve Smith

Stephanie Smits

Elizabeth Smyth

Chi Wai Eric So

Alberto Sobrero

Isabelle Soerjomataram

Payaningal Somanath

M Song

Mingyang Song

Guru Sonpavde

Kjetil Soreide

Henrik Sørensen

L Soroceanu

Robert Soslow

Khalid Sossey-Alaoui

Denis Soulieres

Giulio Spagnoli

Logan G Spector

Valerie Speirs

James Spicer

Salvatore Spicuglia

Philippe E Spiess

Karen-Lise Spindler

Daniel E Spratt

Sanjay K Srivastava

Silvia Stacchiotti

Martin Staege

Margaret Stanley

Naureen Starling

Lesley T Stayner

Justin Stebbing

Nicola Steele

Robert Steele

Stacey Stein

Joachim Steinbach 
Sally Stenning

Robert S Stern

William Steward

Sebastian Stintzing

Martin R Stockler

Melissa Stockwell

Rachael Stolzenberg-Solomon

Michael Stotz

John Strickler

Roger Stupp

Susan R Sturgeon

Erich M Sturgis

Amy Subar

Vivek Subbiah

Patricia Sullivan

Ryan Sullivan

Shi-Yong Sun

Sudha Sundar

Joseph JY Sung

Stéphane Supiot

Tommaso Susini

Jaim Sutton

Hiromu Suzuki

Hiroyoshi Suzuki

Charles Swanton

Dorian Swarts

John Sweetenham

Anthony Swerdlow

Daniel Swinson

Stefan Symeonides

R Paul Symonds

Astri Syse

Peter Szlosarek

Tsutomu Tabata

Josep Tabernero

Pierosandro Tagliaferri

Ayumu Taguchi

David Taieb

Diana Tait

Stephen Tait

Satoshi Takakura

Shigetsugu Takano

Gesche Tallen

Valerie Taly

Rosemary Tambouret

Puay Hoon Tan

Kouichi Tanabe

Shinji Tanaka

Siriwan Tangjitgamol

Coya Tapia

Pedro J Tarraga Lopez

Giulio Tarro

Edward W Tate

Christoph Tausch

Alessandra Tavani

Fattaneh A Tavassoli

Peter M Taylor

Stephen Taylor

Cristina Teixido

Lauren Terras

Krish Tewari

Paul Thelen

Robert Thimme

Fiona Thistlethwaite

Anne Thomas

Daniel Thomas

Alastair Thompson

Deborah Thompson
Fiona Thomson

Ann Thor

Rick F Thorne

David Thwaites

Jaqueline Timmermans

Maria Timofeeva

Jill Tinmouth

Marc Tischkowitz

Apuzzo Tiziana

Thea D Tlsty

Sara Tolaney

Anthony W Tolcher

Rob Tollenaar

Ladislav Tomasek

Iwona M Tomaszewska

Yoshihiko Tomita

Ian PM Tomlinson

$\mathrm{P}$ Tonin

Dott Francesco Torino

Gill Tozer

Tiffany Traina

Ly Thi Hai Tran

Trung Tran

I Trávníček

Franco Trevisani

Cornelia Liu Trimble

Meghana V Trivedi

Jone Trovik

Livio Trusolino

Eric Tse

Konstantinos Tsilidis

Paola Turano

Baris Turkbey

Richard Turkington

Deborah Turnbull

Chris Twelves

Shelley S Tworoger

Jeff Tyner

Toyoaki Uchida

Naoto Ueno

Ilya Ulasov

David Ulmert

Celine Vachon

Ulka Vaishampayan

Claire M Vajdic

LJ Valentijn

ET Valera

Nicola Valeri

Juan Valle

Alexander Van Akkooi

Ann Van den Bruel

Yuri Van der Burgt

Michiel S Van der Heijden

Gabri Van der Pluijm

Tahar Van der Straaten

Pietr Van der Velden

Carla H Van Gils

Jo A Van Ginderachter

Guy Van hazel

Mieke Van Hemelrijck

Jan Van Meerbeeck

Jacco Van Rheenen

Frans Van Roy

Brian Van Tine

Angelo Vanzulli

Janette Vardy

Hebert Alberto Vargas

Judith A Varner
Sophie Vasseur

David Vaughn

Jifke Veenland

Lene Veiga

Guillermo Velasco

Balaji Venugopal

Aldo Venuti

Caroline Verbeke

Freija Verdoodt

Gerald Verhaegh

Henk Verheul

Mukesh Verma

Sten Vermund

Claire Verschraegen

Danny Vesprini

Patrizia Vici

Yana Vinogradova

Tapio Visakorpi

Antonia Vlahou

Kristoffer Von Stedingk

Christian Von Wagner

Elena Voronov

Tom Waddell

Jon Wadsley

Simon Wagner

Zev Wainberg

Richard Wakeford

Max Waldner

Alex Walker

Marjorie Walker

Jo Waller

Matthew Wallis

Paul M Walsh

Fiona Walter

Thomas Walter

$\mathrm{H}$ Wan

Liling Wan

Hong-yang Wang

Jung-Der Wang

Kepeng Wang

Ping Wang

Sophia S Wang

Andrea Wang-Gillam

Jane Wardle

David Warr

Anne Warren

Ruth Warren

Tetsuro Watabe

Justin Waters

David Watkins

David Watson

Valerie Weaver

Arnim Weber

Georg Weber

Jeffrey Weber

Brian D Weiss

James Welsh

Denggui Wen

Nicolas Wentzensen

Haim Werner

S John Weroha

Catharine ML West

Nick West

Shannon Westin

Urban Weyemi

Astrid Weyerbrock

Graham Wheeler

Katriina Whitaker
Jeff White

Gillian Whitfield

A Wicki

Erik Wiemer

Antje Wienecke

DT Wigle

Eva Wikland

Fredrik Wiklund

Gareth Haydn Williams

Stuart Williamson

Gordon Willis

Jeanette Falck Winther

Lori Wirth

T Wisanuyotin

John S Witte

Petronella Witteveen

Isabell Witzel

Dominik Wolf

Federica Wolf

Penella J Woll

Martin Wong

Laura M Woods

Erxi Wu

Ming-Shiang Wu

Wahyu Wulaningsih

David Wynford-Thomas

Ashley Wysong

AM Xavier

Dan Xie

Ren Xu

Rona Yaeger

Akira Yamada

Sohsuke Yamada

Hua Yang

Hushan Yang

Jun Yang

Liying Yang

Qifeng Yang

Timothy A Yap

Yuanqing Ye

I-Tien Yeh

Kam C Yeung

Sai-Ching Yeung

Kein-Leong Yim

Chu-Yiu Yiu

Junji Yodoi

Sam Yoon

Zongbing You

George Yousef

Dominic Yu

Vladimir Yutkin

John Zalcberg

Khalil Zaman

Marco Zappa

AG Zeimet

Baolin Zhang

Fan Zhang

Paul J Zhang

Ruiwen Zhang

Zhuping Zhong

Huiping Zhou

DB Zimmer

Philipp Zimmer

Inti Zlobec

Albert Zlotnik

Manuel Zorzi

Huachun Zou

Jeff I Zwicker 